\title{
Covering the Plane with Two Kinds of Circles*
}

\author{
G. Fejes Tóth
}

Mathematical Institute, Hungarian Academy of Sciences, P.O. Box 127, H-1364 Budapest, Hungary

gfejes@math-inst.hu

Abstract. A sharp lower bound is given for the density of a covering of the plane with two kinds of circles

\section{Introduction}

Let us recall the classical results of Thue [10] and Kershner [8] according to which the density of a packing of equal circles in the plane is at most $\pi / \sqrt{12}$ and the density of a covering of the plane with equal circles is at least $2 \pi / \sqrt{27}$. Both density bounds are sharp and are attained by the incircles and circumcircles of a regular hexagonal tiling, respectively. The book Lagerungen in der Ebene, auf der Kugel und im Raum [5] which laid the foundations for the theory of packing and covering contains a great variety of generalizations of these theorems. As a starting point of the investigations of the present paper, we mention the following result [3], [5, p. 72]:

The density of a packing in the plane consisting of $n$ kinds of circles is at most

$$
1-\left(1-\frac{\pi}{\sqrt{12}}\right)^{n} \text {. }
$$

The geometric meaning of this density bound is the following: If we want to pack the plane possibly densely with $n$ kinds of circles of arbitrary size, then we must pack the plane with unit circles as densely as possible, fill the gaps with very small circles, fill the gaps still remaining with equal circles which are very small compared with the second kind of circles, and so on.

* This research was supported by the Hungarian Foundation for Scientific Research (OTKA) under Grant Numbers 1907 and 14218. 


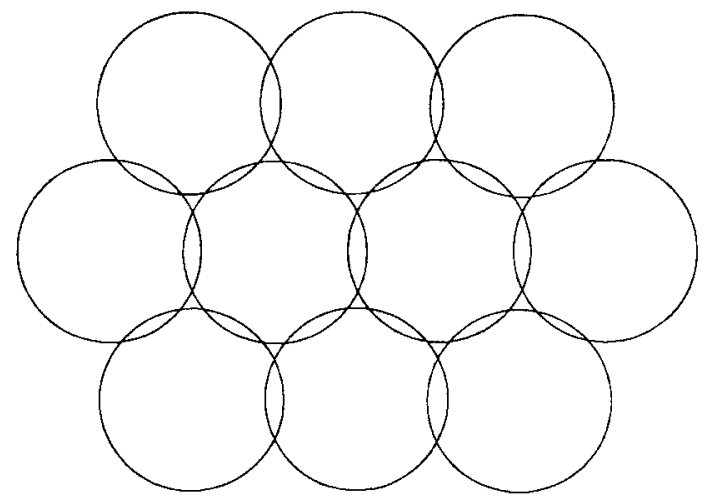

Fig. 1

The dual counterpart of this problem, to cover the plane possibly thinly with two, three, or more kinds of circles, is much more difficult. Here we solve this problem for two kinds of circles.

We use the following notation: int $S$, bd $S$, cl $S$, and conv $S$ denote the interior, boundary, closure, and convex hull of the set $S \in E^{2}$. If $S$ is Lebesque measurable, then its area is denoted by $a(S)$. The cardinality of a set $X$ is denoted by $|X|$.

We consider an arrangement $\mathscr{A}$ of congruent circles concentric with the tiles of a regular hexagonal tiling (see Fig. 1). Let $d$ be the density of $\mathscr{A}$. An easy calculation shows that the quotient of the area of the portion of one hexagon left uncovered by the circles and the area of the hexagon is

$$
\sigma(d)= \begin{cases}1-d & \text { for } 0<d \leq \frac{\pi}{\sqrt{12}}, \\ 1-d+\frac{6 d}{\pi} \arccos \frac{1}{\sqrt[4]{12}} \sqrt{\frac{\pi}{d}} & \text { for } \frac{\pi}{\sqrt{12}}<d \leq \frac{2 \pi}{\sqrt{27}} \\ -\sqrt{\frac{6 \sqrt{3} d-3 \pi}{\pi}} & \text { for } \frac{2 \pi}{\sqrt{27}}<d .\end{cases}
$$

Next we consider a system $\tilde{\mathscr{A}}$ of congruent circles in a regular hexagonal arrangement covering the plane with density $2 \pi / \sqrt{27}$. We obtain a covering with two kinds of circles by supplementing $\mathscr{A}$ by those circles from $\mathscr{A}$ which intersect the part of the plane left uncovered by the circles of $\mathscr{A}$. Choosing the circles of $\tilde{\mathscr{A}}$ sufficiently small compared with those of $\mathscr{A}$, the density of the covering so obtained is arbitrarily close to

$$
d+\frac{2 \pi}{\sqrt{27}} \sigma(d)
$$


Taking the minimum for $d$, we see that there are coverings of the plane with two kinds of circles whose density is arbitrarily close to

$$
\begin{gathered}
d_{2}=\min _{0 \leq d \leq 2 \pi / \sqrt{27}}\left(d+\frac{2 \pi}{\sqrt{27}} \sigma(d)\right)=0.91438 \ldots \\
+\frac{2 \pi}{\sqrt{27}} \sigma(0.91438 \ldots)=1.018955 \ldots
\end{gathered}
$$

On the other hand, we show that any covering of the plane with two kinds of circles has density at least $d_{2}$. This follows immediately from the following:

Theorem. Let $R$ be a rectangle and suppose that some circles of two distinct radii with total area $t$ cover $R$. Then

$$
t \geq d_{2} a(R)
$$

where $d_{2}$ is the constant defined by relations (1) and (2).

A lower bound for the density of a covering with two kinds of circles whose ratio of areas is $1: q, q \geq 1$, was given by Florian [6]. The bound $d_{2}$ is larger than his for $q>26.1$.

Let $d_{i}$ be the infimum of the densities of all coverings of the plane with $i$ kinds of circles. Then, according to Kershner, we have $d_{1}=2 \pi / \sqrt{27}$. Our theorem states that for $i=2$ we have

$$
d_{i}=\min _{0 \leq d \leq 2 \pi / \sqrt{27}}\left(d+d_{i-1} \sigma(d)\right) .
$$

It can be conjectured that this is true for all $i$.

\section{Proof of the Theorem}

We start by collecting some known results used in the proof.

Lemma 1. Let the function $f(q)$ be defined for $q \geq 1$ as

$$
f(q)=\min _{0 \leq m \leq 3,0<l} \frac{2 \pi(m q+l)}{l(6-m) \sin (2 \pi /(6-m))+m(6+l) q \sin (2 \pi /(6+l))} .
$$

If a convex polygon $H$ with at most six sides is covered by circles such that the ratio of areas of any two circles is at most $q$, then the total area of the circles is at least $a(H) f(q)$. 
The proof of a little bit weaker result than Lemma 1 is given in [2] and reproduced on pp. 73-74 of [5]. On p. 79 of [5] the dual counterpart of Lemma 1 for packings is stated explicitly with the note that its proof is immediate. The proof of Lemma 1 is completely analogous. We observe that, in fact, the easy proof yields that it is sufficient to take the minimum for integers $l$ and $m$.

The following result is due to Hadwiger [7] (see also p. 93 of [5] for a proof).

Lemma 2. Any simply connected region with area a and perimeter $p$ can be covered with

$$
\left[\frac{2}{\sqrt{27}} a+\frac{2}{\pi \sqrt{3}} p+1\right]
$$

unit circles.

The following result of L. Fejes Tóth was first proved on pp. 80-84 of [5].

Lemma 3. Let $H$ be a convex polygon with at most six sides and let $C_{1}, \ldots, C_{n}$ be congruent circles of total area $t$. Further, let $U$ be the subset of $H$ which is left uncovered by the circles. Then

$$
a(U) \geq a(H) \sigma\left(\frac{t}{a(H)}\right) .
$$

We need some preparation before the formulation of our last lemma. In what follows it is convenient to regard a half-plane as a circular disk of infinite radius. We consider three closed circular disks, two with unit radius and one with radius $\varrho \geq 1$. We vary the circles under the assumption that their intersection is nonempty and arrange them so that the area of the region covered by at least two of the circles is minimum. It is easily seen that the minimum is attained in the case when the intersection of the circles is a single point and the three chords common to each pair of the circles have equal lengths. The configuration is uniquely determined by this property, and an easy computation gives that, in this case, the area of the region covered by at least two of the circles equals

$$
T(\varrho)=4 \arcsin a+2 \varrho^{2} \arcsin \frac{a}{\varrho}-4 a \sqrt{1-a^{2}}-2 a \sqrt{\varrho^{2}-a^{2}}
$$

where

$$
2 a=\frac{-1+\sqrt{1+8 \varrho^{2}}}{2 \varrho}
$$

denotes the length of a chord common to a pair of circles. 
We have

$$
T(1)=\pi-\frac{\sqrt{27}}{2}=0.543516 \ldots
$$

and

$$
T(\infty)=\pi-2=1.141592 \ldots>2 T(1) .
$$

Further, it is clear from the geometric meaning of $T(\varrho)$ that it is an increasing continuous function. Therefore there is a unique number $\varrho_{0}>1$ such that

$$
T\left(\varrho_{0}\right)=2 T(1)
$$

Numerical calculation shows that

$$
\varrho_{0}=16.7473 \ldots
$$

Now we are ready to formulate our last lemma.

Lemma 4. Let $U$ be a bounded region which is the complement of the union of some circles of radius greater than or equal to $\varrho_{0}$. Suppose that some unit circles of total area $t$ cover $U$ so that the area of each component of $U$ which is covered by a single circle is at most $\sqrt{27} / 2$. Then

$$
t \geq \frac{2 \pi}{\sqrt{27}} a(U)
$$

Lemma 4 generalizes a theorem of L. Fejes Tóth (see [4] and p. 67 of [5] which concerns the case when $U$ is convex. The dual counterpart to our lemma for packings was given by Molnár [9, Theorem 12]. We prove our theorem anticipating the proof of Lemma 4 which we postpone to the next section.

Suppose that the rectangle $R$ is covered by circles of two different kinds, which we refer to as small circles and big circles. We assume without loss of generality that the radius of the small circles is 1 , while the big ones have radius $r>1$. Let $t_{1}$ denote the total area of the big circles, $t_{2}$ the total area of the small circles, and write $t=t_{1}+t_{2}$. If $r<\varrho_{0}$, then, by Lemma 1 ,

$$
\frac{t}{a(R)} \geq f\left(\varrho_{0}^{2}\right)>1.02>d_{2}
$$

Therefore we restrict ourselves to the case when

$$
r \geq \varrho_{0}
$$

Let $U$ be the set of those points of $R$ which are not covered by the big circles. Suppose that $U$ has a connected component $U_{0}$ such that $U_{0}$ is covered by a single small circle and $a\left(U_{0}\right)>\sqrt{27} / 2$. Then we remove this single small circle, together 
with all big circles bounding $U_{0}$. We show that the arising gap can be covered by small circles whose total area is less than that of the removed circles.

$U_{0}$ is bounded by sides of $R$ and by big circles. Let $l_{1}$ and $l_{2}$ be the number of sides of $R$ and the number of big circles bounding $U_{0}$, respectively. We observe that $\sqrt{27} / 2$ is nothing else than the area of a regular hexagon inscribed in a unit circle. Since $U_{0}$ is contained in a unit circle, the assumption $a\left(U_{0}\right)>\sqrt{27} / 2$ readily implies that $l_{1}+l_{2} \geq 7$.

First we consider the case $l_{1}=0$. Then the total area of the removed circles is at least $\left(1+7 r^{2}\right) \pi$. All the removed circles are contained in a circle of radius $1+2 r$. In view of Lemma 2 this circle can be covered by unit circles of total area

$$
\left[\frac{2 \pi}{\sqrt{27}}(1+2 r)^{2}+\frac{4}{\sqrt{3}}(1+2 r)+1\right] \pi .
$$

A simple computation shows that, for $r \geq \varrho_{0}$, this expression is less than $\left(1+7 r^{2}\right) \pi$.

Next let $l_{1}=1$. Now the total area of the removed circles is at least $\left(1+6 r^{2}\right) \pi$. The removed circles are again contained in a circle of radius $1+2 r$, however, since we are interested only in the part of $R$ which gets uncovered, we can decrease this circle by the segment of it cut off by the side of $R$ bounding $U_{0}$. Using the fact that the distance of this side from the center of the circle is at most 2, a rough estimate shows that removing the segment from the circle decreases the area by at least $40 \%$ and the perimeter decreases by at least $10 \%$. Thus, the part of $R$ which gets uncovered is contained in a simply connected region whose area is at most $0.6(1+$ $\left.2 r^{2}\right) \pi$ and whose perimeter is at most $1.8(1+2 r) \pi$. Hence, by Lemma 2 , this part of $R$ can be covered by unit circles of total area

$$
\left[\frac{1.2 \pi}{\sqrt{27}}(1+2 r)^{2}+\frac{2.4}{\sqrt{3}}(1+2 r)+1\right] \pi .
$$

Again, it is easy to check that, for $r \geq \varrho_{0}$, this expression is less than $\left(1+6 r^{2}\right) \pi$. The cases when $l_{1}=2,3$, or 4 can be settled in a similar way. We omit the details.

By the above operation of removing certain circles and replacing them by unit circles, the total area of the circles covering $R$ does not increase. Thus we can assume that the area of no connected component of $U$ which is covered by a single small circle exceeds $\sqrt{27} / 2$.

In view of Lemma 3 we have

$$
a(U) \geq a(R) \sigma\left(\frac{t_{1}}{a(R)}\right)
$$

Further, $U$ and the small circles satisfy the conditions of Lemma 4. Therefore

$$
t_{2} \geq \frac{2 \pi}{\sqrt{27}} a(U)
$$


Combining the last two inequalities and recalling the definition of $d_{2}$ we get

$$
t=t_{1}+t_{2} \geq t_{1}+\frac{2 \pi}{\sqrt{27}} a(R) \sigma\left(\frac{t_{1}}{a(R)}\right) \geq a(R) d_{2} .
$$

This completes the proof of our theorem modulo the proof of Lemma 4 .

\section{Proof of Lemma 4}

We call a set a $Q$-set if it is the complement of the union of some closed circles of radius greater than or equal to $\varrho$. We say that such a set is finitely generated if it the complement of the union of finitely many circles with radius at least $\varrho$. We recall that here a half-plane is considered to be a circle of radius $\infty$.

Let $U$ be a bounded $\varrho_{0}$-set and let $\mathscr{S}$ be a system of closed unit circles covering $U$ so that the area of each connected component of $U$ which intersects a single circle from $\mathscr{S}$ is at most $\sqrt{27} / 2$. Without loss of generality we may assume the following:

(i) $U$ is finitely generated.

(ii) No four centers of circles from $\mathscr{S}$ lie on a circle and no three centers lie on a circle whose center is a boundary point of $U$.

Suppose that for some $C \in \mathscr{S}$ the intersection of $C \cap U$ is not connected. Then there are two circles, say $\tilde{C}_{1}$ and $\tilde{C}_{2}$, from the generating system of $U$ such that $C \cap \tilde{C}_{1} \cap \tilde{C}_{2} \neq \varnothing$ and $C \cap\left(E^{2} \backslash\left(\tilde{C}_{1} \cup \tilde{C}_{2}\right)\right)$ is not connected. Let $a$ and $b$ be the two points of intersection of bd $\tilde{C}_{1}$ and bd $\tilde{C}_{2}$. Let $m$ be the midpoint of the segment $a b$ and let $L$ be the line through $m$ perpendicular to $a b$. It is easily seen that $C$ contains both $a$ and $b$, so it contains $m$ as well. Let $L_{1}$ and $L_{2}$ be the two lines parallel to $L$ at distance 2 from $m$ such that $a$ is between $L$ and $L_{1}$ and $b$ is between $L$ and $L_{2}$. The set $L_{i} \cap\left(E^{2} \backslash\left(\tilde{C}_{1} \cup \tilde{C}_{2}\right)\right)(i=1,2)$ consists of three components: of two rays and a segment. We denote the latter by $S_{i}(i=1,2)$. Clearly, the set $C \cap U$ is contained in the quadrilateral $Q=\operatorname{conv}\left(S_{1} \cup S_{2}\right)$. It is easy to see that the length of $S_{i}$ is at most $2\left(\varrho_{0}-\sqrt{\varrho_{0}^{2}-4}\right)=0.2397 \ldots$, thus $a(Q) \leq 8\left(\varrho_{0}-\sqrt{\varrho_{0}^{2}-4}\right)=0.9588 \ldots<2.598 \ldots=\sqrt{27} / 2$. We modify $\mathscr{S}$ and $U$ by omitting the circle $C$ from $\mathscr{S}$ and by cutting out the set $U \cap Q$ from $U$. The new system $\mathscr{S}$ still covers the new set $U$. Since $a(C) / a(U \cap Q)>\sqrt{27} / 2$, it suffices to prove the inequality $t \geq(\sqrt{27} / 2) a(U)$ for the new $\mathscr{S}$ and $U$.

Our new set $U$ need not be a $\varrho_{0}$-set any longer. However, it remains such a set locally. More precisely, the following condition holds:

(iii) For each circle $C \in \mathscr{S}$ there is a finitely generated $\varrho_{0}$-set $U(C)$ such that $C \cap U=C \cap U(C)$.

The generating set of circles for $U(C)$ is either the same as that for $U$ or is obtained from it by adding one of the half-planes bounded by $L_{1}$ and $L_{2}$ and containing $L$. Since the distance of $L_{1}$ and $L_{2}$ is 4 , it easily follows that:

(iv) For two circles $C_{1}, C_{2} \in \mathscr{S}$ for which $C_{1} \cap C_{2} \neq \varnothing$ we have $U\left(C_{1}\right)=U\left(C_{2}\right)$. 
Now we can repeat the above operation so that properties (iii) and (iv) are preserved until the intersection of $U$ with each circle from $\mathscr{S}$ is connected. Thus it suffices to prove the lemma in the case when:

(v) For each circle $C \in \mathscr{S}$ the intersection $C \cap U=C \cap U(C)$ is connected.

In view of (v) we can partition $\mathscr{S}$ into subclasses so that the circles from one subclass intersect the same connected component of $U$. Thus it suffices to prove the lemma for connected sets $U$. Henceforth, we assume that:

(vi) $U$ is connected.

Let $C_{1}, \ldots, C_{n}$ be the circles from $\mathscr{S}$. Without loss of generality we assume that:

(vii) No proper subset of $\mathscr{S}$ covers $U$.

The validity of the lemma is supposed for $n=1$, so we have to consider only the case when

$$
n \geq 2 \text {. }
$$

Let $D_{1}, \ldots, D_{n}$ be the Dirichlet cells of the circles $C_{1}, \ldots, C_{n}$, i.e., $D_{i}(i=1, \ldots, n)$ consists of those points of the plane which are closer to the center of $C_{i}$ than to the center of any circle $C_{j}, j=1, \ldots, n, j \neq i$. The sets $D_{i}, i=1, \ldots, n$, are mutually disjoint and their closures cover the plane. The Dirichlet cell of $C_{i}$ with respect to $U$ is the set $D_{i} \cap U$. Since the circles $C_{1}, \ldots, C_{n}$ cover $U$, we have

$$
D_{i} \cap U \subset C_{i}
$$

for all $i=1, \ldots, n$. Further, in view of (vii), $D_{i} \cap \neq \varnothing$ for $i=1, \ldots, n$. The set $D_{i} \cap U$ need not be connected. Next we describe a procedure which associates to each set $D_{i} \cap U$ which is not connected, a connected set containing it.

Let $U$ be a $\varrho$-set and let $C$ be a circle whose radius is less than $\varrho$. Suppose that $U \cap C$ is connected, and observe that then $U \cap C$ is simply connected. Moreover, if $C \cap U \neq U$, then each component of $C \backslash U$ is simply connected. In this case, if $p$ and $q$ are points of bd $U$ belonging to the same component of $\operatorname{cl}(C \backslash U)$, then there is a well-defined arc of bd $U$ connecting $p$ to $q$ inside $C$.

Now, let $D$ be a polygon such that $D \cap U$ is contained in $C$. Suppose that $D \cap U$ is not connected. Then we associate to $D \cap U$ a connected set $\hat{D}$ as follows:

We observe that $\operatorname{bd}(D \cap C)$ falls into arcs lying inside and outside $U$ alternately following each other. We consider the arcs lying outside $U$. If such an arc connects the point $p$ to $q$, then we replace it by the arc of bd $U$ connecting $p$ to $q$ inside $C$. In this way we obtain a closed continuous curve which we denote by $B$. Some points of $B$ are visited twice, however, $B$ does not cross itself. Now the set $\hat{D}=(D \cap U)$ $\cup B$ is connected and its boundary is exactly the curve $B$.

If an arc of bd $U$ is visited twice by the curve $B$, then we think of the two arcs of $B$ as being separated by an infinitesimal corridor. Thus we think of $\hat{D}$ as of topological disks (see Fig. 2).

Let $\hat{D}_{i}$ be the set obtained by applying the above procedure to the sets $D_{i}, C_{i}$, and $U\left(C_{i}\right)$ in the place of $D, C$, and $U$. The new sets $\hat{D}_{i}, i=1, \ldots, n$, will still form a 


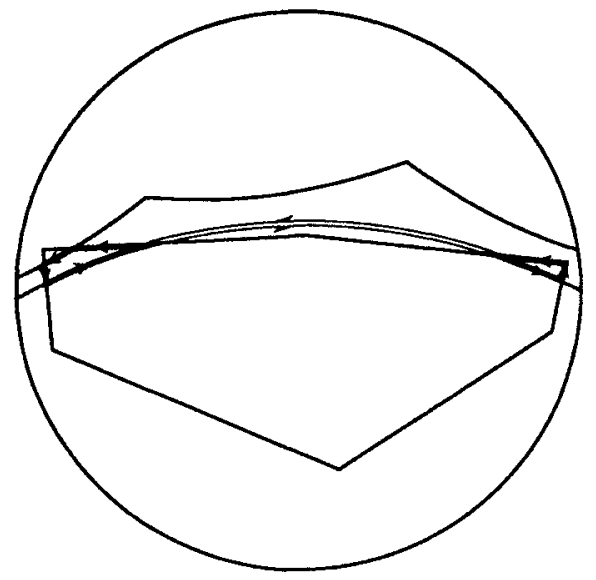

Fig. 2

packing. Let $\hat{D}_{n+1}, \ldots, \hat{D}_{N}(N>n)$ be the connected components of the complement of $U$. Since $U$ is connected and bounded, with the exception of one unbounded component, all components of its complement are simply connected. We consider the cell complex $\mathscr{E}$ whose faces are $\hat{D}_{1}, \ldots, \hat{D}_{N}$. An edge $e$ of $\mathscr{E}$ is a maximal arc common to two faces, say $\hat{D}_{i}$ and $\hat{D}_{j}$, of $\mathscr{E}$. A vertex of $\mathscr{E}$ is the common endpoint of some edges of $\mathscr{C}$. The set of edges and vertices of $\mathscr{C}$ are denoted by $E$ and $V$, respectively.

Assumption (ii) guarantees that in all vertices of $\mathscr{C}$ exactly three edges meet. Hence it follows immediately by Euler's theorem that $|V|=2(N-2)$. Since $N>n$, we have

$$
|V| \geq 2 n-2 .
$$

An edge which is common to the cells $\hat{D}_{i}$ and $\hat{D}_{j}$ with $i, j \leq n$ is called internal, otherwise it is called external. A vertex is called internal if all edges meeting in it are internal and it is called external otherwise. It is easily seen that in each external vertex exactly one internal and two external edges meet. We denote by $V_{\mathrm{i}}$ and $V_{\mathrm{e}}$ the set of internal and external vertices, respectively.

With each edge $e \in E$ we associate a set $L(e)$ as follows: Let $e$ be an edge common to $\hat{D}_{i}$ and $\hat{D}_{j}$. If $i, j \leq n$, then let $L(e)=C_{i} \cap C_{j}$. If, on the other hand, $i \leq n$ and $j>n$, then let $L(e)$ be the connected component of $C_{i} \cap \hat{D}_{j}$, the boundary of which contains $e$. For a vertex $v \in V$ in which the edges $e_{1}, e_{2}$, and $e_{3}$ meet, we define $I(v)$ as the connected component of $L\left(e_{1}\right) \cap L\left(e_{2}\right) \cap L\left(e_{3}\right)$ which contains $v$. We write

$$
t(v)=a\left(L\left(e_{1}\right)\right)+a\left(L\left(e_{2}\right)\right)+a\left(L\left(e_{3}\right)\right)-2 a(I(v)) .
$$

We note that in the case when $v$ is an internal vertex common to the cells $\hat{D}_{i}, \hat{D}_{j}$, and $\hat{D}_{k}$, with $i, j, k \leq n$, then $t(v)$ is nothing else than the area of the part of the plane covered at least twice by the circles $C_{i}, C_{j}$, and $C_{k}$. Thus we have

$$
t(v)>T(1)=\pi-\frac{\sqrt{27}}{2} \quad \text { if } \quad v \in V_{\mathrm{i}} .
$$


Let $v$ be an external vertex of $\mathscr{E}$ in which the cells $\hat{D}_{i}, \hat{D}_{j}$, and $\hat{D}_{k}$ meet so that $i, j \leq n$ and $k>n$. Let $e_{1}, e_{2}$, and $e_{3}$ be the edges of $\mathscr{E}$ emanating from $v$ so that $e_{1}$ is common to $\hat{D}_{i}$ and $\hat{D}_{j}, e_{2}$ is common to $\hat{D}_{i}$ and $\hat{D}_{k}$, and, finally, $e_{3}$ is common to $\hat{D}_{j}$ and $\hat{D}_{k}$. Since $C_{i} \cap C_{j} \neq \varnothing$, we have, by $(\mathrm{v}), U\left(C_{i}\right)=U\left(C_{j}\right)$. Let $\tilde{C}$ be a circle from the generating system of $U\left(C_{i}\right)=U\left(C_{j}\right)$ such that $v \in \operatorname{cl} \tilde{C}$. Then we have $L\left(e_{2}\right) \subset C_{i} \cap \tilde{C}$ and $L\left(e_{3}\right) \subset C_{j} \cap \tilde{C}$. Hence it easily follows that $t(v)$ cannot be less than the area of the part of the plane covered at least twice by the circles $C_{i}, C_{i}$, and $\tilde{C}$. Thus we have

$$
t(v) \geq T\left(\varrho_{0}\right)=2 T(1) \quad \text { if } \quad v \in V_{\mathrm{e}} .
$$

The main tool to the proof of Lemma 4 is the following:

Proposition 1. We have

$$
a(U)=n \pi-\frac{1}{2} \sum_{v \in V} t(v) .
$$

For the case when $U$ is convex this formula was proved in [4]. A similar formula for the area of a convex disk covered by $n$ translates of a centrally symmetric convex disk has been proved in [1].

Before proceeding with the proof of Proposition 1, we show that it readily implies Lemma 4. In view of the assumption $n \geq 2$ we have $\left|V_{\mathrm{e}}\right| \geq 2$. Thus, using Proposition 1 and inequalities (3)-(5) we get

$$
a(U)=n \pi-\frac{1}{2} \sum_{v \in V} t(v) \leq n \pi-\frac{1}{2}\left(\left|V_{\mathrm{i}}\right| T(1)+\left|V_{\mathrm{e}}\right| 2 T(1)\right) \leq n \pi-n T(1)=\eta \frac{\sqrt{27}}{2} .
$$

We consider the circle $C_{i}(i \leq n)$ and the cell $\hat{D}_{i}$ associated with it. The edges of $\mathscr{C}$ bounding $\hat{D}_{i}$ are called the sides of $\hat{D}_{i}$. We assign a positive orientation so that the interior of $\hat{D}_{i}$ is on the left while travelling on bd $\hat{D}_{i}$ in the positive direction. Let $v_{1}, \ldots, v_{r}$ be the vertices of $\mathscr{C}$ lying on the boundary of $\hat{D}_{i}$ enumerated in the positive cyclic order and write $v_{r+1}=v_{1}$. Let $s_{m}$ denote the side of $\hat{D}_{i}$ connecting $v_{m}$ to $v_{m+1}$.

With each side $s_{m}, m=1, \ldots, r$, we associate an arc $s_{m}^{*}$ which is obtained by elongating $s_{m}$ in both directions to the boundary of $C_{i}$. We call this arc the chord of $C_{i}$ determined by $s_{m}$. The way of elongation depends on the type of $s_{m}$ and its vertices.

If $s_{m}$ is an external edge of $\mathscr{C}$, then let $s_{m}^{*}$ be the connected component of $C_{i} \cap$ bd $U$ containing $s_{m}$.

Next consider the case when $s_{m}$ is an internal edge of $\mathscr{E}$ which is common to $\hat{D}_{i}$ and $\hat{D}_{j}(i, j \leq n)$. Let $p_{m}$ and $q_{m}$ be the points of intersection of bd $C_{i}$ and bd $C_{j}$ so that $D_{i}$ is on the left-hand side of the oriented line $p_{m} q_{m}$. We describe how $s_{m}$ is elongated beyond $v_{m}$; the process for $v_{m+1}$ is similar. If $v_{m}$ is an external vertex of $\mathscr{E}$, then it lies on the segment $p_{m} q_{m}$. However, if it is an internal vertex, then it may occur that it does not lie on $p_{m} q_{m}$ but on an arc of bd $U$ (Fig. 3). In this latter case the segment $p_{m} q_{m}$ intersects the component of $C_{i} \cap$ bd $U$ containing $v_{m}$. Then, in the first step of the construction, we elongate $s_{m}$ beyond $v_{m}$ through the correspond- 


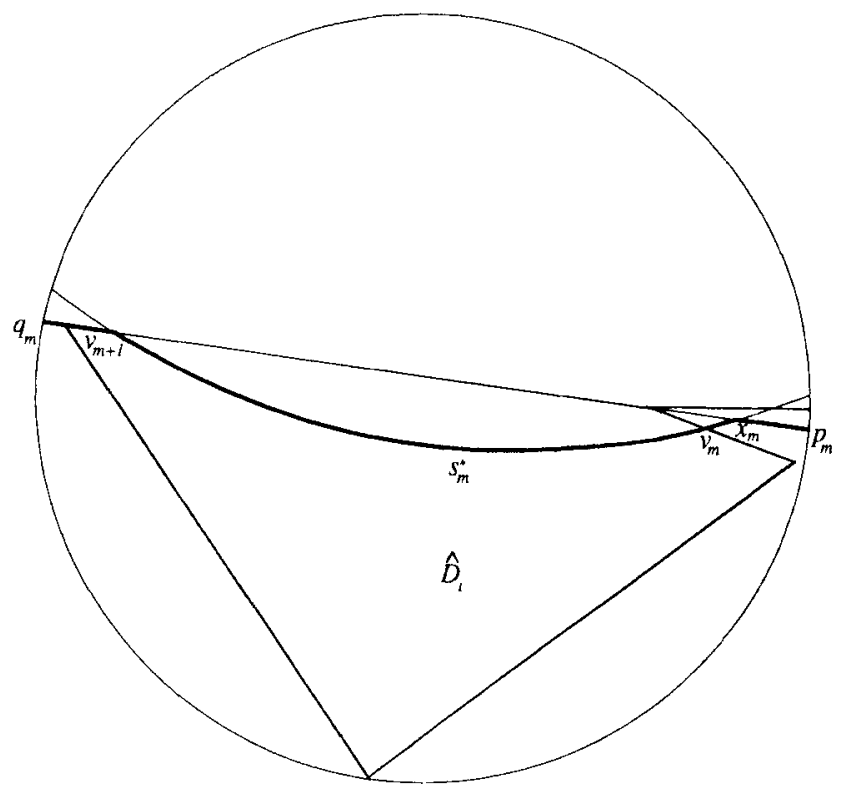

Fig. 3

ing arc of $C_{i} \cap$ bd $U$ to the first point of intersection with the segment $p_{m} q_{m}$. Let us denote this point by $x_{m}$. Further, write $x_{m}=v_{m}$ if $v_{m} \in p_{m} q_{m}$.

Now we define the arc of $s_{m}^{*}$ between $p_{m}$ and $x_{m}$. This is done as follows: We travel on $p_{m} q_{m}$ from $p_{m}$ in the direction of $q_{m}$. If we arrive at a point $y \in \operatorname{bd} U$ such that the connected component of $C_{i} \cap$ bd $U$ intersects the segment of $x_{i} y$ in a point different from $y$, then we travel along this arc to the last point of intersection with $p_{m} q_{m}$ before $x_{m}$. From here on we travel further on $p_{m} q_{m}$ and repeat the procedure above, if necessary. In finitely many steps we arrive at $x_{m}$.

The chord $s_{m}^{*}$ divides $C_{i}$ into two connected components; the one which is disjoint from int $\hat{D}_{i}$ is denoted by $S_{m}$ and is called the segment of $C_{i}$ cut off by $s_{m}^{*}$.

We note that if $s_{m}$ is an external edge of $\mathscr{C}$, then we have $S_{m}=L\left(s_{m}\right)$. Further, if $e$ is an edge of $\mathscr{C}$ common to $\hat{D}_{i}$ and $\hat{D}_{j}$, then the chords of $C_{i}$ and $C_{j}$ determined by $e$ coincide. Furthermore, the union of the segments of $C_{i}$ and $C_{j}$ cut off by $e^{*}$ is just the set $L(e)$.

Let $a_{1}$ be an arc contained in $C_{i}$ and let $a_{2}$ be a subarc of $s_{m}^{*}(1 \leq m \leq r)$. We say that $a_{1}$ crosses the chord $a_{2}$ if $a_{1} \cap a_{2} \neq 0$ and $a_{1}$ contains points both in int $S_{m}$ and in $\operatorname{int}\left(C_{i} \backslash S_{m}\right)$. We say that $a_{1}$ crosses $a_{2}$ more than once if $a_{1}$ contains two disjoint subarcs both crossing $a_{2}$.

Let $s_{m}^{+}$and $s_{m}^{-}$be the subarcs of $s_{m}^{*}$ from $v_{m}$ through $v_{m+1}$ to $q_{m}$ and from $p_{m}$ through $v_{m}$ to $v_{m+1}$, respectively. The construction of the chords $s_{m}^{*}$ readily implies the following properties:

(viii) For every pair $j, k(1 \leq j \leq k \leq r)$ the arcs $s_{j}^{-}$and $s_{k}^{-}$do not cross and neither do the arcs $s_{j}^{+}$and $s_{k}^{+}$. Furthermore, the arcs $s_{j}^{-}$and $s_{k}^{+}$, as well as the arcs $s_{j}^{+}$and $s_{k}^{-}$, cross at most once. 
We have

$$
a\left(D_{i} \cap U\right)=a\left(\hat{D}_{i}\right)=a\left(C_{i}\right)-a\left(\bigcup_{m=1}^{r} S_{m}\right) \quad(i=1, \ldots, n) .
$$

Writing $N(r)=\{1, \ldots, r\}$ we have

$$
a\left(\bigcup_{m=1}^{r} S_{m}\right)=\sum_{m=1}^{r}(-1)^{m-1} \sum_{\substack{M \in N(r) \\|M|=m}} a\left(\bigcap_{j \in M} S_{j}\right)
$$

Let us suppose that for some $j$ and $k$ we have $S_{j} \cap S_{k} \neq \varnothing$. Let $S$ be a connected component of $S_{j} \cap S_{k}$. Then property (viii) implies that $S$ is bounded either by a subarc of $s_{j}^{+}$, by a subarc of $s_{k}^{-}$, and an arc of bd $C_{i}$, or it is bounded by a subarc of $s_{j}^{-}$, by a subarc of $s_{k}^{+}$, and an arc of bd $C_{i}$. Without loss of generality we assume the first case. Let $s_{l}$ be a side of $\hat{D}_{i}$ which lies between $s_{j}$ and $s_{k}$ when we go around bd $\hat{D}_{i}$ in the positive direction. It is easily seen that the points $p_{j}, p_{l}, p_{k}, q_{j}$, $q_{l}, q_{k}$ lie on bd $C_{i}$ in the cyclic order as enumerated here. Since by (viii) the chord $s_{l}^{*}$ does not cross the arcs $s_{j}^{+}$and $s_{k}^{-}$, it follows that

$$
S \subset S_{l}
$$

Suppose that there are $t$ sides of $D_{i}$ between $s_{j}$ and $s_{k}$. Then there are $\left(\begin{array}{c}t \\ m-2\end{array}\right)$ possibilities of obtaining $S$ as the intersection of $m$ different segments of $C_{i}$. This gives a contribution to the sum on the right-hand side of (7) equal to

$$
a(S) \sum_{m=2}^{t+2}(-1)^{m-1}\left(\begin{array}{c}
t \\
m-2
\end{array}\right)
$$

This term is equal to $-a(S)$ if $t=0$, that is, if $s_{j}$ and $s_{k}$ are adjacent sides of $D_{i}$, and it is 0 otherwise. Thus, writing $S_{0}=S_{r}$ and denoting by $J_{m}$ the connected component of $S_{m-1} \cap S_{m}$ containing the vertex $v_{m}$, we have

$$
a\left(\bigcup_{m=1}^{r} S_{m}\right)=\sum_{m=1}^{r}\left(a\left(S_{m}\right)-a\left(J_{m}\right)\right)
$$

Hence we obtain, by (6),

$$
a\left(D_{i} \cap U\right)=a\left(\hat{D}_{i}\right)=\pi-\sum_{m=1}^{r}\left(a\left(S_{m}\right)-a\left(J_{m}\right)\right) \quad(i=1, \ldots, n)
$$


Summing up this for $i=1, \ldots, n$ we get

$$
a(U)=\sum_{i=1}^{n} a\left(D_{i} \cap U\right)=n \pi-\sum_{e \in E} a(L(e))+\sum_{v \in V} a(I(v)) .
$$

From here Proposition 1 follows by observing that

$$
\sum_{e \in E} a(L(e))-\sum_{v \in V} a(I(v))=\frac{1}{2} \sum_{v \in V} t(v) .
$$

\section{Acknowledgment}

I thank Aladár Heppes for valuable remarks.

\section{References}

1. G. Fejes Tóth, Finite covering by translates of centrally symmetric convex domains, Discrete Comput. Geom. 2 (1987), 353-363.

2. L. Fejes Tóth, Einige Bemerkungen über die dichteste Lagerung inkongruenter Kreise, Comment. Math. Helv. 17 (1944-45), 256-261.

3. L. Fejes Tóth, Ausfüllung eines konvexen Bereiches durch Kreise, Publ. Math. Debrecen 1 (1949), 92-94.

4. L. Fejes Tóth Über die dichteste Kreislagerung und dünnste Kreisüberdeckung, Comment. Math. Helv. 23 (1949), 342-349.

5. L. Fejes Töth, Lagerungen in der Ebene, auf der Kugel und im Raum, Springer-Verlag, Berlin, 19532 edn., 1972.

6. A. Florian, Überdeckungen der Ebene durch Kreise, Rend. Sem. Mat. Univ. Padova 31 (1961), 77-86.

7. H. Hadwiger, Überdeckungen ebener Bereiche durch Kreise und Quadrate, Comment. Math. Helv. 13 (1940-1941), 195-200.

8. R. Kershner, The number of circles covering a set, Amer. J. Math. 61 (1939), 665 671.

9. J. Molnár, Packings of circles on surfaces of constant curvature (in Hungarian), Magyar Tud. Akad. Mat. Fiz. Oszt. Közl. 12 (1962), 223-263.

10. A. Thue, Über die dichteste Zusammenstellung von kongruenten Kreisen in der Ebene, Christiania Vid. Slsk. Skr. 1 (1910), 3-9.

Received March 4, 1994, and in revised form September 30, 1994. 